\begin{tabular}{|c|l|}
\hline Title & A method for structuring stochastic travel time by using risk premiums of stochastic link flow \\
\hline Author(s) & Tani, Ryuichi; Kato, Teppei; Uchida, Kenetsu \\
\hline Citation & $\begin{array}{l}\text { Transportmetrica. A, Transport science, 17(2), 212-232 } \\
\text { https://doi.org/10.1080/23249935.2020.1776416 }\end{array}$ \\
\hline Issue Date & 2021 \\
\hline Doc URL & http://hdl.handle.net/2115/83732 \\
\hline Rights & $\begin{array}{l}\text { This is an A ccepted Manuscript of an article published by Taylor \& Francis in Transportmetrica A: Transport Science } \\
\text { on 2021, available online: http:/Www.tandfonline.com/10.1080/23249935.2020.1776416. }\end{array}$ \\
\hline Type & article (author version) \\
\hline File Information & Draft22.pdf \\
\hline
\end{tabular}

Instructions for use 


\section{A method for structuring stochastic travel time by using risk premiums of stochastic link flow}

Ryuichi, Tani* ${ }^{\mathrm{a} *}$, Teppei, Kato ${ }^{\mathrm{b}}$ and Kenetsu, Uchida ${ }^{\mathrm{c}}$

${ }^{a}$ Graduate School of Engineering, Hokkaido University, Sapporo, Japan; ${ }^{b}$ Graduate School of Science and Engineering, Saitama University, Saitama, Japan; ' School of Engineering, Hokkaido University, Sapporo, Japan

Email: ryuichitani@eis.hokudai.ac.jp 


\title{
A method for structuring stochastic travel time by using risk premiums of stochastic link flow
}

\author{
This study proposes a method for structuring stochastic link travel times in a road \\ network with stochastic traffic demands. In our proposed method, the uncertainty \\ of travel time is evaluated by link-flow-based indices. The mean link travel time is \\ represented by using both its mean link flow and the corresponding risk premium \\ of the stochastic link flow. The risk premium is defined by a concept of certainty \\ equivalent derived from the relationship between a stochastic link flow and a link \\ cost function. By expanding the concept of risk premium, a calculation method of \\ link travel time covariance is proposed. Our proposed method defines bi-variate \\ risk premiums, by which the mean of the product of two stochastic link travel times \\ can be calculated. As a numerical calculation, we demonstrate our proposed \\ method in a test network. Finally, we conclude and show future directions for \\ evaluating real road networks.
}

Keywords: stochastic traffic demand; travel time reliability; risk premium; certainty equivalent

Subject classification codes: include these here if the journal requires them

\section{Introduction}

Day-to-day travel time observations reveal stochastic fluctuation of travel time in road networks. Some empirical studies have indicated that travel time uncertainty affects a driver's route choice behavior (Abdel-Aty et al., 1997). For this reason, travel time uncertainty has increasingly drawn attention in transportation research.

One of the main problems for road administrators is how they evaluate the uncertainty of travel time in road networks for evaluating the quality of transport projects. Recently, several papers have proposed traffic assignment models considering some kinds of uncertainties in a road network, so-called reliability-based user equilibrium (RUE) model (e.g., Lo \& Tung, 2003.; Lo et al.; 2006; Shao et al., 2006; Watling, 2006; Lam et al., 2008; Siu \& Lo, 2008; Chen \& Zhou., 2010; Wang et al., 2014). Some of these 
studies assumed no correlation on link travel times because of large computational burdens (e.g., Lo et al., 2006; Shao et al., 2006; Chen \& Zhou., 2010; Wang et al., 2014). However, empirical studies have shown that ignoring the correlation of travel time can result in an inaccurate evaluation of transport projects (e.g., Nicholson, 2015; Nicholson \& Watling, 2015). Following this context, some papers have proposed RUE models with correlated link travel time. (e.g., Prakash et al., 2018) These models are difficult to be solved because these models are often formulated as path-based RUE models, which have many variables and have a non-unique solution. Although algorithms for solving RUE models with correlated link travel time have been proposed (e.g. Srinivasan et al., 2014), there is still no algorithm that is enough efficient to be solved for practical use as far as the authors know.

In the case of analyzing connectivity or vulnerability of road networks, it is necessary to forecast travel time in a road network with link disruptions. Jenelius et al. (2006) have proposed link importance indices and exposure indices based on the generalized travel cost without considering congestions caused by link disruptions. They indicated that the consideration of the congestions is important in vulnerability analysis. For considering congestions in vulnerability analysis, traffic simulations or traffic assignments must be performed for each link disruption. For example, Xu et al. (2018) have proposed a bi-level programming model, in which the upper level is network capacity maximizing problem and the lower level is traffic assignment problem, for addressing the measure of network redundancy. In these models, the impact of travel demand fluctuation is not considered because of the large computational burden, because RUE models with correlated link travel times are difficult to be solved by why we mentioned above. However, correlation of link travel times can be considered as an 
important factor for considering link disruptions, because a link disruption affects widespread road networks.

Some papers have proposed network design problems and road pricing problems with travel time reliability. Lo \& An (2015) and Uchida et al. (2015) have proposed reliability-based network design problems (RNDP) that minimize the sum of the expected total travel time and the other costs, i.e., operational cost; Di et al. (2018) have proposed RNDP that maximizes the flow-based accessibility; Sumalee \& Xu (2011) have proposed a first best marginal cost pricing problem that minimizes the weighted sum of mean and variance of total travel time. As shown above, many kinds of criteria have been used for measuring network performance. On the other hand, drivers' disutility for transportation is used for the criteria in the context of cost-benefit analysis. From the perspective of general users, the optimal design of a road network should be performed by maximizing the user benefits among the whole road network. Therefore, it is reasonable to adopt the driver's disutility as a criterion for measuring network performance for RNDP. Clark \& Watling (2005) proposed a method that calculates the stochastic distribution of total travel time by using the multivariate moments of the stochastic link flows. However, it is still difficult to calculate total drivers' disutility for uncertain travel time by their method.

For overcoming above mentioned difficulties, this study proposes a simple method that calculates a driver's disutility for stochastic travel time in the presence of correlation of link travel time. We adopted the concepts of certainty equivalent and risk premium in financial economics in our proposed method. By following concepts of certainty equivalent and risk premium, the driver's disutility for stochastic travel time is defined by using mean and risk premiums of the stochastic link flow. For considering the correlation of link travel times, the conventional definitions of certainty equivalent and risk premium are extended in this study. Accordingly, our proposed method decomposes 
the covariance of link travel time into two components of corresponding two links. Thus, we define contributions for driver's disutility of each link in a road network by applying our proposed method. They enable us to analyze the driver's disutility for stochastic travel time in the presence of correlation of link travel time by using mean and risk premiums of the stochastic link flow.

The outline of this paper is as follows. In section 2, stochastic traffic flows and stochastic travel times are formulated, and approximated representations of mean and variance-covariance of stochastic link travel times are also shown. In section 3 , the model proposed in section 2 is demonstrated by using both a simple situation and a test network. In section 4 , we conclude and show future perspectives.

\section{Formulation}

In this section, we formulate stochastic traffic flow, mean and variance-covariance of stochastic link travel time based on the certainty-equivalent concept. Note that variables in capital letters and those in lower case letters are random variables and deterministic variables, respectively. A notation list is summarized in Appendix A.

\subsection{Traffic flow}

This study assumes stochastic traffic demands. Note that, the discussion in this subsection is independent of the distribution type of a stochastic origin-destination (OD) traffic demand. However, additive random variables such as a normal distribution should be selected as OD traffic demands, when the same theoretical distribution among OD traffic demands, path flows and link flows is required. Mean of traffic demand between OD pair $w$ is given as $q_{w}=E\left[Q_{w}\right]$ where $Q_{w}$ is the stochastic OD demand. When the coefficient of variation is given as $c v_{w}$, a variance of OD traffic demand is defined as $\operatorname{var}\left[Q_{w}\right]=$ $\left(c v_{w} \cdot q_{w}\right)^{2}$. By summing up the all stochastic path flows between OD pair $w, F_{k}^{w}$, OD 
demand $Q_{w}$ is represented as

$$
Q_{w}=\sum_{k \in K_{w}} F_{k}^{w} \quad \forall w \in W
$$

Stochastic link flow $V_{a}$ is represented as a summation of all path flows which pass through link $a$.

$$
V_{a}=\sum_{w \in W} \sum_{k \in K_{w}} \delta_{w, k, a} \cdot F_{k}^{w} \quad \forall a \in A
$$

where $\delta_{w, k, a}$ is a variable that equals one if path $k$ between OD pair $w$ passes link $a$, and zero otherwise. Mean of OD traffic flow between OD pair $w, q_{w}$ is the summation of all mean path flows between the OD pair $w, f_{k}^{w}$. The mean path flow is always nonnegative.

$$
\begin{gathered}
q_{w}=\sum_{k \in K_{w}} f_{k}^{w} \quad \forall w \in W \\
f_{k}^{w} \geq 0 \quad \forall k \in K_{w}, w \in W
\end{gathered}
$$

where $f_{k}^{w}=E\left[F_{k}^{w}\right]$. Mean link flow $v_{a}=E\left[V_{a}\right]$ is the summation of the mean of path flows which pass through link $a$.

$$
v_{a}=\sum_{w \in W} \sum_{k \in K_{w}} \delta_{w, k, a} \cdot f_{k}^{w} \quad \forall a \in A
$$

\subsection{Certainty equivalent of traffic flow}

In this subsection, we apply concepts of certainty equivalent and risk premium developed in financial economics to the formulation of stochastic travel times in a road network. For a simple explanation, we assume a random variable $X$ and a utility function $u(X)$. Then, the expected utility is represented as 


$$
E[u(X)]=u\left(x_{o}\right)
$$

where $x_{o}$ is a certainty equivalent of a random variable, $X$, with respect to a utility function, $u(X)$. In other words, the certainty equivalent of a random variable $X$ is a deterministic variable specific to the expected utility. We apply this relationship to the representation of link travel time and link flow. Based on our interpretation, stochastic link flow and link cost function correspond to random variable $X$ and utility function $u(X)$, respectively. Note that, this study adopts BPR function (Bureau of Public Roads, 1964) as a link cost function:

$$
t_{a}\left(v_{a}\right)=t_{a}^{o} \cdot\left(1+\alpha_{a} \cdot\left(\frac{v_{a}}{c_{a}}\right)^{n_{a}}\right) \quad \forall a \in A
$$

where $c_{a}$ and $t_{a}^{o}$ are link traffic capacity and free-flow travel time. $\alpha_{a}$ and $n_{a}$ in (7) are calibration parameters. In general, the BPR function is applied to a deterministic road network where traffic flows and travel times are represented by deterministic variables. However, in this study, by substituting a stochastic link flow $V_{a}$ for $v_{a}$ to BPR function, we define stochastic link travel time as a random variable following previous studies such as Lam et al. (2008) and Uchida (2014).

$$
t_{a}\left(V_{a}\right)=t_{a}^{o} \cdot\left(1+\alpha_{a} \cdot\left(\frac{V_{a}}{c_{a}}\right)^{n_{a}}\right) \quad \forall a \in A
$$

We understand that some studies assume that a traffic demand does not follow a normal distribution and that a link travel time follow some specific distribution such as a lognormal distribution (e.g. Sumalee \& Xu, 2011; Chen et al., 2018; Tani \& Uchida, 2018) and a Burr distribution (e.g. Susilawati et al., 2013; Taylor, 2017). When a 
stochastic link flow $V_{a}$ is separated by the deterministic term $v_{a}$ and random term $\varepsilon_{a}$, risk premium of link flow $\pi_{a}$ is defined by using mean and certainty equivalent of link flow.

$$
\pi_{a}=v_{a}^{o}-v_{a} \quad \forall a \in A
$$

where $v_{a}^{o}$ is the certainty equivalent of link flow. By using deterministic risk premium of link flow, the mean link travel time is redefined as

$$
E\left[t_{a}\left(v_{a}+\varepsilon_{a}\right)\right]=t_{a}\left(v_{a}+\pi_{a}\right) \quad \forall a \in A
$$

Then, first-ordered Taylor series approximations of both sides of (10) are respectively represented as

$$
\begin{gathered}
E\left[t_{a}\left(v_{a}+\varepsilon_{a}\right)\right] \approx E\left[t_{a}\left(v_{a}\right)+\frac{1}{2} t_{a}^{(2)}\left(v_{a}\right) \cdot \varepsilon_{a}^{2}\right]=t_{a}\left(v_{a}\right)+\frac{1}{2} t_{a}^{(2)}\left(v_{a}\right) \cdot \operatorname{var}\left[V_{a}\right] \\
t_{a}\left(v_{a}+\pi_{a}\right) \approx t_{a}\left(v_{a}\right)+t_{a}^{(1)}\left(v_{a}\right) \cdot \pi_{a} \quad \forall a \in A(11) \quad \forall a \in A
\end{gathered}
$$

Note that $t_{a}^{(n)}$ in (11) is $n$-th order derivative of a link cost function with respect to mean link flow $v_{a}$. It is not necessary to consider high-dimensional terms more than twodimensional terms in (11), because we assume that link flow follows a normal distribution. Higher-dimensional moments than two-dimensional terms of a normal distribution are negligibly small. We also assume that the right side of (10) is linearly approximated as (12). From the above two approximated equations, a linear equation with respect to the risk premium of mean link flow, $\pi_{a}$, is explicitly deducted. By solving (10)-(12), risk premium of link flow, $\pi_{a}$, is defined as an approximated closed form: 


$$
\pi_{a}=\frac{1}{2} \frac{t_{a}^{(2)}\left(v_{a}\right)}{t_{a}^{(1)}\left(v_{a}\right)} \operatorname{var}\left[V_{a}\right] \quad \forall a \in A
$$

Following (9) and (13), the certainty equivalent of link flow is represented by using mean and risk premium of link flow shown as

$$
v_{a}^{o}=v_{a}+\pi_{a}=v_{a}+\frac{1}{2} \frac{t_{a}^{(2)}\left(v_{a}\right)}{t_{a}^{(1)}\left(v_{a}\right)} \operatorname{var}\left[V_{a}\right] \quad \forall a \in A
$$

The certainty equivalent of link flow is determined by parameters of a link cost function, mean and variance of link flow. The discussion above is based on the results shown in Pratt (1964). Note that risk premium of link flow, $\pi_{a}$, converges to zero when link flow variance approaches to zero, or equivalently link flow changes to deterministic variable shown as

$$
\lim _{\operatorname{var}\left[V_{a}\right] \rightarrow 0} \pi_{a}=0 \forall a \in A
$$

A risk premium of link flow which is defined in (13) can be regarded as the effect of the random term of link flow on a link cost function. Figure 1 shows an intuitive explanation of the relationship between the mean of link travel time and the certainty equivalent of link flow. In Figure 1, link travel time of link $a, t_{a}\left(v_{a}\right)$, is shown when mean link flow is $v_{a}=1,000[\mathrm{PCU} /$ hour $]$. The mean of link travel time, $E\left[t_{a}\left(V_{a}\right)\right]$, is slightly larger than $t_{a}\left(v_{a}\right)$. Here, certainty equivalent of link flow, $v_{a}^{o}$, can be calculated subject to $E\left[t_{a}\left(V_{a}\right)\right]=t_{a}\left(v_{a}^{o}\right)$. And thus, the risk premium of link flow, $\pi_{a}$, corresponds to additional link flow in terms of the mean of link travel time.

[Figure 1 near here] 
In general, in financial economics, for deduction of risk premium based on Pratt (1964) from the relationship between utility function and stochastically-distributed price of a good, either of following two conditions must be satisfied, i.e., either (i) price of good follows elliptical distribution (e.g. Samuelson, 1970; Chamberlain, 1983; Owen and Rabinovitch, 1983) or (ii) utility function is quadratic (e.g. Tobin, 1958). It is unrealistic to assume a quadratic link cost function. Thus, this study assumes stochastic link flow following an elliptical distribution. Many prior studies, e.g. Shao et al. (2006)., Lam et al. (2008)., Uchida (2014), assume that link flows follow normal distributions, which is a typical example of elliptical distributions. Assumption of normal distribution makes it easy to formulate traffic flow modeling, due to its reproductive property. In the following discussion, we assume normally distributed link flows.

\subsection{Variance-covariance of stochastic link travel time}

In this section, we define bi-variate risk premiums of two link flows for calculating the mean value of the product of two stochastic link travel times. A set of the bi-variate risk premiums, i.e., $\hat{\pi}_{a \mid b}$ and $\hat{\pi}_{b \mid a}$, satisfy the following equation:

$$
E\left[t_{a}\left(v_{a}+\varepsilon_{a}\right) \cdot t_{b}\left(v_{b}+\varepsilon_{b}\right)\right]=t_{a}\left(v_{a}+\hat{\pi}_{a \mid b}\right) \cdot t_{b}\left(v_{b}+\hat{\pi}_{b \mid a}\right) \quad \forall a \in A, b \in A
$$

Therefore, the covariance of link travel time can be formulated as

$$
\operatorname{cov}\left[T_{a}, T_{b}\right]=t_{a}\left(v_{a}+\hat{\pi}_{a \mid b}\right) \cdot t_{b}\left(v_{b}+\hat{\pi}_{b \mid a}\right)-t_{a}\left(v_{a}+\pi_{a}\right) \cdot t_{b}\left(v_{b}+\pi_{b}\right)(17)
$$

When covariance of two link travel times, $\operatorname{cov}\left[T_{a}, T_{b}\right]$, is given, a vector of bi-variate risk

premiums, $\left(\hat{\pi}_{a \mid b}, \hat{\pi}_{b \mid a}\right)^{T}$ in the above equation is not specified uniquely, because every point on the nonlinear curve shown by (17) can be the bi-variate risk premiums. In some studies of financial economics, the same discussions have been provided. Duncan (1977) 
proposed a method for specifying risk premiums vector by linear approximation of utility function. An example obtained by applying the method of Duncan (1977) to this study is shown in Appendix B.

The method proposed in Duncan (1977) cannot decompose covariance of link travel time into risk factors that are stochastic link flows. Therefore, we made some assumptions on a set of bi-variate risk premiums. Risk-premium-based link travel time covariance shown in (17) is transformed as

$$
\begin{aligned}
\operatorname{cov}\left[T_{a}, T_{b}\right] & =\left(t_{a}\left(v_{a}+\hat{\pi}_{a \mid b}\right)+t_{a}\left(v_{a}+\pi_{a}\right)\right) \cdot\left(t_{b}\left(v_{b}+\hat{\pi}_{b \mid a}\right)-t_{b}\left(v_{b}+\pi_{b}\right)\right) \\
& -t_{a}\left(v_{a}+\pi_{a}\right) \cdot t_{b}\left(v_{b}+\hat{\pi}_{b \mid a}\right)+t_{a}\left(v_{a}+\hat{\pi}_{a \mid b}\right) \cdot t_{b}\left(v_{b}+\pi_{b}\right)
\end{aligned}
$$

As shown above, the covariance of link travel time can be decomposed into a "product" term shown by the first term and a "cross" term shown by the second and third terms of the right-hand side on (18). If bi-variate risk premiums vector, $\left(\hat{\pi}_{a \mid b}, \hat{\pi}_{b \mid a}\right)^{T}$, is defined so that

$$
t_{a}\left(v_{a}+\pi_{a}\right) \cdot t_{b}\left(v_{b}+\hat{\pi}_{b \mid a}\right)-t_{a}\left(v_{a}+\hat{\pi}_{a \mid b}\right) \cdot t_{b}\left(v_{b}+\pi_{b}\right)=0 \quad \forall a, b \in A
$$

can hold, the covariance of link travel time is then represented only as

$$
\operatorname{cov}\left[T_{a}, T_{b}\right]=\left(t_{a}\left(v_{a}+\hat{\pi}_{a \mid b}\right)+t_{a}\left(v_{a}+\pi_{a}\right)\right) \cdot\left(t_{b}\left(v_{b}+\hat{\pi}_{b \mid a}\right)-t_{b}\left(v_{b}+\pi_{b}\right)\right)
$$

In this case, (19) can be regarded as an identity that constrains the feasible region defined by (17). For explaining the necessity of the identity, (19), we can describe the geometrical meaning of the relationship of (17), (19) and (20) by using Figure 2. Geometrically, a covariance of two different link travel times, $\operatorname{cov}\left[T_{a}, T_{b}\right]$ is the striped area enclosed by 
points $A, B, C, D, E$ and $F$ in Figure 2. The condition shown by (19) means the region represented by $\overrightarrow{O C}=m \cdot \overrightarrow{O F}$ where $m \geq 1.0$. The feasible region for point $C$ is defined by (17) and is shown by a bold solid line segment in Figure 2. This condition finds a solution as the intersection between the feasible region, (17) and the line defined by (19).

While Figure 2 describes the link-travel-time-based geometrical representation, Figure 3 describes the link-flow-based geometrical representation. The intersection between (17) and (20) in Figure 3 corresponds to that in Figure 2. Figure 2 can be interpreted as a mapping of Figure 3 with respect to a link cost function. Note that the solution of the pair of risk premiums by the method of Duncan (1977) is obtained by solving the problem: $\arg \min \hat{\pi}_{a \mid b}^{2}+\hat{\pi}_{b \mid a}^{2}$. This is a norm minimization problem constraining to (17). The detail is described in the latter of this section.

[Figure 2 near here]

[Figure 3 near here]

The theoretical definition of covariance of link travel time may not be convenient for numerical calculation. Therefore, we use the approximated expression of link travel time given by (20). First-ordered Taylor series approximations of both sides of (16) respectively are

$$
\begin{gathered}
E\left[t_{a}\left(v_{a}+\varepsilon_{a}\right) \cdot t_{b}\left(v_{b}+\varepsilon_{b}\right)\right] \approx t_{a}\left(v_{a}\right) \cdot t_{b}\left(v_{b}\right)+\frac{1}{2} \operatorname{tr}\left(\mathbf{U}_{a, b} \mathbf{\Sigma}_{a, b}\right) \quad \forall a \in A, b \in A \\
t_{a}\left(v_{a}+\hat{\pi}_{a}\right) \cdot t_{b}\left(v_{b}+\hat{\pi}_{b}\right) \approx t_{a}\left(v_{a}\right) \cdot t_{b}\left(v_{b}\right)+\mathbf{u}_{a, b}^{T} \hat{\pi}_{a, b} \forall a \in A, b \in A
\end{gathered}
$$

where

$$
\mathbf{u}_{a, b}=\nabla\left(t_{a}\left(v_{a}\right) \cdot t_{b}\left(v_{b}\right)\right)=\left[\begin{array}{c}
t_{a}^{(1)}\left(v_{a}\right) \cdot t_{b}\left(v_{b}\right) \\
t_{a}\left(v_{a}\right) \cdot t_{b}^{(1)}\left(v_{b}\right)
\end{array}\right] \quad \forall a \in A, b \in A
$$




$$
\begin{gathered}
\widehat{\boldsymbol{\pi}}_{a, b}=\left[\begin{array}{l}
\hat{\pi}_{a \mid b} \\
\hat{\pi}_{b \mid a}
\end{array}\right] \quad \forall a \in A, b \in A \\
\mathbf{U}_{a, b}=\nabla^{2}\left(t_{a}\left(v_{a}\right) \cdot t_{b}\left(v_{b}\right)\right)=\left[\begin{array}{cc}
t_{a}^{(2)}\left(v_{a}\right) \cdot t_{b}\left(v_{b}\right) & t_{a}^{(1)}\left(v_{a}\right) \cdot t_{b}^{(1)}\left(v_{b}\right) \\
t_{b}^{(1)}\left(v_{b}\right) \cdot t_{a}^{(1)}\left(v_{a}\right) & t_{a}\left(v_{a}\right) \cdot t_{b}^{(2)}\left(v_{b}\right)
\end{array}\right] \quad \forall a \in A, b \in A \\
\boldsymbol{\Sigma}_{a, b}=\left[\begin{array}{cc}
\operatorname{var}\left[V_{a}\right] & \operatorname{cov}\left[V_{a}, V_{b}\right] \\
\operatorname{cov}\left[V_{a}, V_{b}\right] & \operatorname{var}\left[V_{b}\right]
\end{array}\right] \quad \forall a \in A, b \in A
\end{gathered}
$$

In (23) and (25), $\nabla$ is an operator of partial differential defined as $\left[\partial / \partial v_{a}, \partial / \partial v_{b}\right]^{T}$. In the same manner as (10)-(12), since the high-dimensional terms more than two dimensional terms are negligibly small, we performed the first-order Taylor series expansion to both sides of (16). Following Duncan (1977), a set of bi-variate risk premiums of two link flows is given as the solution of

$$
\mathbf{u}_{a, b}^{T} \widehat{\boldsymbol{\pi}}_{a, b}-\frac{1}{2} \operatorname{tr}\left(\mathbf{U}_{a, b} \boldsymbol{\Sigma}_{a, b}\right)=0 \quad \forall a \in A, b \in A
$$

A set of risk premiums is not yet specified in the above equation, (27). Duncan (1977) specified it by using a generalized inverse matrix. As shown in Figure 3, their method minimizes the norm between the origin point and the solution set represented by equation (27) geometrically. This method is reasonable even if we get a tentative solution, but there is no theoretical detailed background to interpret the obtained pair of risk premiums. Then, we specify a set of bi-variate risk premiums with reasonable geometric interpretation in our proposed model. We solve a nonlinear simultaneous equation system shown by (19) and (27). Thus, the variance-covariance matrix of link travel time is simply represented as

$$
\boldsymbol{\Sigma}_{\mathrm{T}}=\boldsymbol{X}_{\mathrm{T}}^{+} \circ \boldsymbol{X}_{\mathrm{T}}^{+}
$$


where

$$
\begin{gathered}
\mathbf{X}_{\mathbf{T}}^{+}=\left[\begin{array}{ccc}
X_{1 \mid 1}^{+} & \cdots & X_{1|| A \mid}^{+} \\
\vdots & \ddots & \vdots \\
X_{|A| \mid 1}^{+} & \cdots & X_{|A||| A \mid}^{+}
\end{array}\right] \\
\mathbf{X}_{\mathbf{T}}^{-}=\left[\begin{array}{ccc}
X_{1 \mid 1}^{-} & \cdots & X_{|A| \mid 1}^{-} \\
\vdots & \ddots & \vdots \\
X_{1|| A \mid}^{-} & \cdots & X_{|A||| A \mid}^{-}
\end{array}\right] \\
X_{a \mid b}^{+}=t_{a}\left(v_{a}+\hat{\pi}_{a \mid b}\right)+t_{a}\left(v_{a}+\pi_{a}\right) \forall a, b \in A \\
X_{a \mid b}^{-}=t_{a}\left(v_{a}+\hat{\pi}_{a \mid b}\right)-t_{a}\left(v_{a}+\pi_{a}\right) \forall a, b \in A
\end{gathered}
$$

Note that $A \circ B\left(\forall A, B \in \mathbb{R}^{n \times n}\right)$ implies a Hadamard product of two matrices $A$ and $B$. Thus, each element of the variance-covariance matrix is represented as a "product" of two risk components, i.e., $X_{a \mid b}^{+}$and $X_{b \mid a}^{-}$(or, $X_{a \mid b}^{-}$and $\left.X_{b \mid a}^{+}\right) \quad(\forall a, b \in A)$. This form means that covariance of link travel time could be represented as an area of the rectangle of which two sides are given by $X_{a \mid b}^{+}$and $X_{b \mid a}^{-}$, or $X_{a \mid b}^{-}$and $X_{b \mid a}^{+}$.

We structure risk premium and bi-variate risk premiums, which corresponding to mean and mean of product of two stochastic link travel times as above. By structuring the risk premiums and bi-variate risk premiums, the risk of the whole road network can be measured by link-based indices. In a field of financial risk management, portfolio risk depends on the variation of risk factors. Our study assumes that portfolio risk and risk factors correspond to stochastic total travel time and stochastic traffic flows, respectively. The detail of this discussion is described in Appendix B.

By using risk premiums and bi-variate risk premiums defined above, mean and variance-covariance of link travel times and mean and variance of path travel times are respectively defined as 


$$
\begin{gathered}
E\left[T_{a}\right]=t_{a}\left(v_{a}+\pi_{a}\right) \quad \forall a \in A \\
\operatorname{cov}\left[T_{a}, T_{b}\right]=t_{a}\left(v_{a}+\hat{\pi}_{a \mid b}\right) \cdot t_{b}\left(v_{b}+\hat{\pi}_{b \mid a}\right)-t_{a}\left(v_{a}+\pi_{a}\right) \cdot t_{b}\left(v_{b}+\pi_{b}\right) \quad \forall a, b \in A \\
E\left[T_{w, k}\right]=\sum_{k \in K_{w}} \delta_{w, k, a} \cdot E\left[T_{a}\right] \quad \forall k \in K_{w}, \forall w \in W \\
\operatorname{var}\left[T_{w, k}\right]=\operatorname{var}\left[\sum_{k \in K_{w}} \delta_{w, k, a} \cdot T_{a}\right] \\
=\sum_{a \in A} \sum_{b \in A} \delta_{w, k, a} \cdot \delta_{w, k, b} \cdot \operatorname{cov}\left[T_{a}, T_{b}\right] \forall k \in K_{w}, \forall w \in W
\end{gathered}
$$

\section{Numerical calculation}

\subsection{In a small example}

For demonstrating our proposed method, we show some results of numerical calculations in a small example. As a simple example, we set a mean link flow vector and variancecovariance matrix as $\mathbf{v}=[1000,800]^{T}$ and $\boldsymbol{\Sigma}_{\mathbf{v}}=c v^{2}$.

$\left[\begin{array}{cc}1000^{2} & \rho \cdot 1000 \cdot 800 \\ \rho \cdot 1000 \cdot 800 & 800^{2}\end{array}\right]$, respectively. Note that the coefficient of variation, $c v$ and the coefficient of correlation, $\rho$, are set as 0.5 and 0.2 , respectively. Calibration parameters of two BPR functions, $t_{0 i}, \alpha, n$ and $c_{i}(i \in\{1,2\})$ are set as $0.05,2,6$ and 1000 , respectively. A set of risk premiums corresponding to the mean of link travel times is calculated as $\boldsymbol{\pi}^{T}=(80,100)^{T}$.

[Figure 4 near here]

Figure 4 shows three domains (or curves) for feasible sets of risk premiums shown by (17), (19) and (26). Solid line (1) in Figure 3 is the curve shown by (17). Two dotted lines, (2) and (3), are curves shown by (19) and (27), respectively. The set of bi-variate risk premiums is specified by solving a nonlinear simultaneous equation system which is 
composed of (19) and (27). For example, the set of bi-variate risk premiums corresponding to $\operatorname{cov}\left[T_{1}, T_{2}\right]$ or $\operatorname{cov}\left[T_{2}, T_{1}\right]$ is calculated as $\hat{\boldsymbol{\pi}}=[97.3$, $113.6]^{T}$ by solving a nonlinear simultaneous equation system shown by (19) and (27). We obtain the variance-covariance matrix of link travel time, and corresponding two matrices, $\mathbf{X}_{\mathbf{T}}^{+}$and $\mathbf{X}_{\mathbf{T}}^{-}$respectively shown as

$$
\begin{gathered}
\boldsymbol{\Sigma}_{\mathbf{T}}=\left[\begin{array}{ll}
0.0023 & 0.0027 \\
0.0027 & 0.0508
\end{array}\right] \\
\mathbf{X}_{\mathbf{T}}^{+}=\left[\begin{array}{ll}
X_{1 \mid 1}^{+} & X_{1 \mid 2}^{+} \\
X_{2 \mid 1}^{+} & X_{2 \mid 2}^{+}
\end{array}\right]=\left[\begin{array}{ll}
0.2044 & 0.1986 \\
0.4679 & 0.5471
\end{array}\right] \\
\mathbf{X}_{\mathbf{T}}^{-}=\left[\begin{array}{ll}
X_{1 \mid 1}^{-} & X_{2 \mid 1}^{-} \\
X_{1 \mid 2}^{-} & X_{2 \mid 2}^{-}
\end{array}\right]=\left[\begin{array}{ll}
0.0115 & 0.0136 \\
0.0058 & 0.0928
\end{array}\right]
\end{gathered}
$$

The variance-covariance of link travel time in (37) is calculated by Taylor's approximation by using the right sides of (11) and (21), respectively. We can calculate the results of (38) and (39) directly by using (31), (32) and $\widehat{\boldsymbol{\pi}}$ specified above. Just for the reference, the set of bi-variate risk premiums which is specified by the above method is shown as a cross mark in Figure 4.

\subsection{In a road network}

We demonstrate our proposed model in the test network of Nguen and Dupuis (1984). In the network, we calculate the vector of risk premiums and the matrix of bi-variate risk premiums, respectively. The test network in Figure 5 is composed of four OD pairs, 25 paths and 19 links. The OD pairs are listed as $(1,2),(1,3),(4,2)$ and $(4,3)$. Because of space limitations, sets of the path-link sequence are omitted (See Tani and Uchida, 2018).

[Figure 5 near here] 
Mean traffic demand and its coefficient of variation of each OD pair are set as 1,000 [PCU/hour] and 0.2, respectively. All calibration parameters of the BPR function are the same as those of the first experiment. Here, we formulate a logit-based stochastic traffic assignment model. The path choice probabilities are determined by a logit model so that they are discrete random variables. Some studies such as Nakayama and Watling (2014) discuss the stochasticity of the path choice probability. Their study considers the stochasticity of the path flows by generating the samples of traffic flows from the path choice probability. In other words, the path choice probability is regarded as continuous random variables. However, we regard it as discrete random variables. This assumption is the same as the many previous studies such as Chen et al. (2006), Lam et al. (2008) and Tani and Uchida (2018) for the simplicity of the numerical calculation. This is because the results of the numerical calculation for this proposed method are independent no matter whether the path choice probability is deterministic or not. The calculated risk premiums following the proposed method are determined only by the mean and variancecovariance of link flows and the shape of a link cost function.

The link-path sequence is fixed, and four OD traffic demands are assigned to 25 paths. SUE traffic assignment model is formulated as a fixed-point problem shown as

$$
\mathbf{f}_{w}=q_{w} \cdot \mathbf{p}_{w}\left(\boldsymbol{\eta}_{w}(\mathbf{f})\right) \quad \forall w \in W
$$

where

$$
\begin{gathered}
p_{k}^{w}=\frac{\exp \left(-\theta \cdot \eta_{k}^{w}\right)}{\sum_{k \in \mathbf{K}_{w}} \exp \left(-\theta \cdot \eta_{k}^{w}\right)} \quad \forall k \in K, \forall w \in W \\
\mathbf{f}_{w}=\left(f_{w, 1}, \cdots, f_{w,\left|\mathbf{K}_{w}\right|}\right) \quad \forall w \in W \\
\mathbf{f}=\left(\mathbf{f}_{1}, \cdots, \mathbf{f}_{|W|}\right) \quad \forall w \in W
\end{gathered}
$$




$$
\begin{aligned}
& \mathbf{p}_{w}=\left(p_{w, 1}, \cdots, p_{w,\left|K_{w}\right|}\right) \quad \forall w \in W \\
& \boldsymbol{\eta}_{w}=\left(\eta_{w, 1}, \cdots, \eta_{w,\left|K_{w}\right|}\right) \quad \forall w \in W
\end{aligned}
$$

Note that the dispersion parameter of the driver's utility in (41), $\theta$ is set as 1 . The traffic assignment problem is solved by the path-based algorithm, the method of successive analysis (MSA) following Sheffi (1985). In stochastic road networks, drivers choose their paths based on not only mean but also a variance of path travel times. Thus, we set driver's disutility function shown as

$$
\eta_{w, k}=E\left[T_{w, k}\right]+\gamma \cdot \operatorname{var}\left[T_{w, k}\right] \quad \forall k \in K_{w}, \forall w \in W
$$

A risk-aversion parameter in (46), $\gamma$ is set as 2 . When the parameter, $\gamma$ is equal to zero, the driver's path choice behavior is risk-neutral. The parameter $\gamma$ is interpreted as a calibrating coefficient converting variance of travel time into disutility. This assumption of disutility function follows the discussion in Fosgerau and Engelson (2011).

As calculation results, we show a variance-covariance matrix of link travel time. Figures 6 and 7 show the two variance-covariance matrices of link flows calculated by (26) and link travel times calculated by (28), respectively. Figures 8 and 9 show the risk component matrices, $\mathbf{X}_{\mathbf{T}}^{+}$and $\mathbf{X}_{\mathbf{T}}^{-}$defined in (28)-(32).

[Figure 6 near here]

[Figure 7 near here]

[Figure 8 near here]

[Figure 9 near here]

As shown in Figures 8 and 9, the relative trend between $\mathbf{X}_{\mathbf{T}}^{+}$and a transpose of $\mathbf{X}_{\mathbf{T}}^{-}$are the same as each other, because $\mathbf{X}_{\mathbf{T}}^{+}-\mathbf{t} \mathbf{1}^{T}$ is equal to $\mathbf{X}_{\mathbf{T}}^{-T}+\mathbf{t} \mathbf{1}^{T}$ following (31) and (32). Note that $\mathbf{t}$ represents a column vector of the mean of link travel time, and $\mathbf{1}$ represents a 
column vector whose components are all ones. If we summarize the $\mathbf{X}_{\mathrm{T}}^{+}-\mathbf{t} \mathbf{1}^{T}$ or $\mathbf{X}_{\mathrm{T}}^{-T}+$ $\mathbf{t 1}^{T}$ along the row, we obtain a vector that represents the sum of the travel time of each link that corresponds to a component of a variance-covariance matrix of link travel time. Figure 10 represents the vector. Each component of the vector is represented as $\sum_{a^{\prime} \in A} t_{a}\left(v_{a}+\hat{\pi}_{a \mid a^{\prime}}\right) a \in A$, respectively.

[Figure 10 near here]

Both matrices, $\mathbf{X}_{\mathbf{T}}^{+}-\mathbf{t} \mathbf{1}^{T}$ and $\mathbf{X}_{\mathbf{T}}^{-T}+\mathbf{t} \mathbf{1}^{T}$ are the same as each other from the definition of (31) and (32). In other words, $\mathbf{X}_{\mathbf{T}}^{+}-\mathbf{t} \mathbf{1}^{T}$ and $\mathbf{X}_{\mathbf{T}}^{-T}+\mathbf{t} \mathbf{1}^{T}$ are parallelly translated by the constant vectors, $2 \mathbf{t} \mathbf{1}^{T}$ and $-2 \mathbf{t} \mathbf{1}^{T}$, respectively. Besides, the relative order of each component of the vector shown in Figure 10 and that of the mean link travel time, $\mathbf{t}$ are closely similar to each other, because the ratio between $t_{a}\left(v_{a}+\hat{\pi}_{a \mid a^{\prime}}\right)$ and $t_{a^{\prime}}\left(v_{a^{\prime}}+\right.$ $\left.\hat{\pi}_{a^{\prime} \mid a}\right)$ is determined by the corresponding mean link travel times following (19).

\section{Conclusion}

This study proposes a simplified method for structuring the mean and covariance of stochastic link travel time. We introduce conventional concepts of certainty equivalent and risk premium, originally developed in financial economics, to the representation of mean and covariance of stochastic link travel times. When considering stochastic traffic demands in a conventional framework, both link flows and link travel times are represented as multivariate random variables in general. By the proposed method, mean and covariance of link travel times are respectively represented by corresponding risk premiums and bi-variate risk premiums of link flows. Those risk premiums and bi-variate risk premiums of stochastic link flows are defined by parameters of link cost functions and variations of link flows. Especially, the covariance of two link travel times can be decomposed by two risk components. Each risk component attributes to each stochastic 
link flow. Thus, we can evaluate the uncertainty of link travel time of the whole road network based on the newly developed indices defined in this study.

We demonstrated the method proposed in this study. The results show that our proposed method decomposes total travel time variance (or risk of total travel time) in a network into the risk of each link. The risk allocated to each link is interpreted as a covariate effect of total travel time variance. We also show the way to understand the relative importance of each link in terms of the risk premiums matrices corresponding to a variance-covariance matrix of link travel times. By using the risk component matrices, we obtain the indicators that represent the relative contribution of the travel time reliability of each link to the variability of the whole road network.

As future tasks, the proposed method could be applied to a network design problem with equilibrium constraints on uncertain travel time and stochastic traffic flow. Besides, the application of our proposed method to a real road network is also needed.

\section{Acknowledgments}

This study was supported by the Committee on Advanced Road Technology (CART), Ministry of Land, Infrastructure, Transport, and Tourism, Japan.

\section{References}

Abdel-Aty, M. A., Kitamura, R., and Jovanis, P. P. 1997. "Using stated preference data for studying the effect of advanced traffic information on drivers' route choice.", Transportation Research Part C: Emerging Technologies 5(1): 39-50. doi: https://doi.org/10.1016/S0968-090X(96)00023-X

An, K., and Lo, H. K. 2015 "Robust transit network design with stochastic demand considering development density, Transportation Research Part B: Methodological." 81: 737-754. doi: https://doi.org/10.1016/j.trb.2015.05.019

Bureau of Public Roads. 1964. Traffic Assignment Manual. U.S. Department of Commerce, Urban Planning Division, Washington DC. 
Chamberlain, G. 1983. "A characterization of the distributions that imply mean-variance utility functions." Journal of Economic Theory 29: 185-201. doi: https://doi.org/10.1016/0022-0531(83)90129-1

Chen, A., Yang, C., Kongsomsaksakul, S., and Lee, M. 2006. "Network-based accessibility measures for vulnerability analysis of degradable transportation networks." Networks and Spatial Economics 7(3): 241-256. Doi: https://doi.org/10.1007/s11067-006-9012-5

Chen, A., and Zhou, Z. 2010. "The $\alpha$-reliable mean-excess traffic equilibrium model with stochastic travel times." Transportation Research Part B: Methodological 44(4): 493-513. Doi: https://doi.org/10.1016/j.trb.2009.11.003

Chen, P., Tong, R., Lu, G., and Wang, Y. 2018. "The $\alpha$-reliable path problem in stochastic road networks with link correlations: A moment-matching-based path finding algorithm." Expert Systems with Applications 115: 20-32. Doi: https://doi.org/10.1016/j.eswa.2018.05.022

Clark, S., and Watling, D. 2005. "Modelling network travel time reliability under stochastic demand." Transportation Research Part B: Methodological 39(2): 119-140. Doi: https://doi.org/10.1016/j.trb.2003.10.006

Di, Z., Yang, L., Qi, J., and Gao, Z. 2018. “Transportation network design for maximizing flow-based accessibility." Transportation Research Part B: Methodological 110: 209-238. Doi: https://doi.org/10.1016/j.trb.2018.02.013

Duncan, G. T. 1977. "A matrix measure of multivariate local risk aversion." Econometrica 45(4): 895-903. doi: https://www.jstor.org/stable/1912680

Fosgerau, M., and Engelson, L. 2011. “The value of travel time variance.” Transportation Research Part B: Methodological 45(1): 1-8.

doi: https://doi.org/10.1016/j.trb.2010.06.001

Jenelius, E., Petersen, T., and Mattsson, L. G. 2006. "Importance and exposure in road network vulnerability analysis." Transportation Research Part A: Policy and Practice 40(7): 537-560. doi: https://doi.org/10.1016/j.tra.2005.11.003

Lam, W.H.K., Shao, H., and Sumalee, A. 2008. "Modeling impacts of adverse weather conditions on a road network with uncertainties in demand and supply." Transportation Research Part B: Methodological, 42(10): 810-890. doi: https://doi.org/10.1016/j.trb.2008.02.004 
Lo, H. K., and Tung, Y. K. 2003. "Network with degradable links: Capacity analysis and design." Transportation Research Part B: Methodological 37(4): 345-363. doi: https://doi.org/10.1016/S0191-2615(02)00017-6

Lo, H. K., Luo, X. W., and Siu, B. W. Y. 2006. "Degradable transport network: Travel time budget of travelers with heterogeneous risk aversion." Transportation Research Part B: Methodological 40: 792-806. doi: https://doi.org/10.1016/j.trb.2005.10.003

Nakayama, S., and Watling, D. 2014. "Consistent formulation of network equilibrium with stochastic flows." Transportation Research Part B: Methodological 66: 5069. doi: https://doi.org/10.1016/j.trb.2014.03.007

Nguyen, S. and Dupuis, C. 1984. "An efficient method for computing traffic equilibria in networks with asymmetric transportation costs." Transportation Science 18(2): 185-202. doi: https://doi.org/10.1287/trsc.18.2.185

Nicholson, A., and Watling, D. 2015. "Modelling issues in incorporating link travel time correlations in the analysis of corridor trip reliability." Proceedings of the 6th International Symposium on Transportation Network Reliability.

Nicholson, A. 2015. “Travel time reliability benefits: Allowing for correlation.” Research in Transportation Economics 49: 14-21. doi: https://doi.org/10.1016/j.retrec.2015.04.002

Owen, J., and Rabinovitch, R. 1983. "On the class of elliptical distributions and their applications to the theory of portfolio choice." The Journal of Finance 38(3):745752. doi: https://www.jstor.org/stable/2328079

Prakash, A. A., Seshadri, R., and Srinivasan, K. K. 2018 "A consistent reliability-based user-equilibrium problem with risk-averse users and endogenous travel time correlations: Formulation and solution algorithm." Transportation Research Part B: Methodological 114: 171-198. doi: https://doi.org/10.1016/j.trb.2018.06.003

Pratt, J. W. 1964. "Risk aversion in the small and in the large." Econometrica 32: 122136. doi: https://www.jstor.org/stable/1913738

Samuelson, P. A. 1970. "The fundamental approximation theorem of portfolio analysis in terms of means, variances and higher moments." The Review of Economic Studies 37(4): 537-542. doi: https://www.jstor.org/stable/2296483

Shao, H., Lam, W. H. K., and Tam, M. L. 2006. "A reliability-based stochastic traffic assignment model for network with multiple user classes under uncertainty in 
demand." Networks and Spatial Economics 6(34): 173-204. Doi: https://doi.org/10.1007/s11067-006-9279-6

Sheffi, Y. 1985. Urban Transportation Networks: Equilibrium analysis with mathematical programming methods. Prentice-Hall, Englewood Cliffs, New Jersey.

Siu, B. W. Y., and Lo, H. K. 2008. "Doubly uncertain transportation network: Degradable capacity and stochastic demand." European Journal of Operational Research 191(1): 164-179. doi: https://doi.org/10.1016/j.ejor.2007.08.026

Srinivasan, K. K., Prakash, A. A., and Seshadri, R. 2014. "Finding most reliable paths on networks with correlated and shifted log-normal travel times." Transportation Research Part B: Methodological 66: 110-128. doi: https://doi.org/10.1016/j.trb.2013.10.011

Sumalee, A., and Xu, W. 2011. "First-best marginal cost toll for a traffic network with stochastic demand." Transportation Research Part B: Methodological 45(1): 4159. doi: https://doi.org/10.1016/j.trb.2010.04.007

Susilawati, S., Taylor, M. A. P., and Somenahalli, S. V. C. 2013. "Distributions of travel time variability on urban roads." Journal of Advanced Transportation 47: 720730. doi: https://doi.org/10.1002/atr.192

Taylor, M. A. P. 2017. "Fosgerau's travel time reliability ratio and the Burr distribution." Transportation Research Part B: Methodological 97: 50-63. doi: https://doi.org/10.1016/j.trb.2016.12.001

Tani, R., Owada, T., and Uchida, K. 2020. "Path travel time estimation method by incomplete traffic data." International Journal of Intelligent Transportation Systems Research 18: 43-52. doi: https://doi.org/10.1007/s13177-018-0168-4

Tani, R., and Uchida, K. 2018. “A stochastic user equilibrium assignment model under stochastic demand and supply following lognormal distribution." Asian Transportation Studies 5 (2): 326-348. doi: https://doi.org/10.11175/eastsats.5.326

Tobin, J. E. 1958. "Liquidity preference as behavior towards risk." Review of Economic Studies 25: 65-86. doi: https://www.jstor.org/stable/2296205

Uchida, K. 2014. "Estimating the value of travel time and of travel time reliability in road networks." Transportation Research Part B: Methodological 66: 129-147. doi: https://doi.org/10.1016/j.trb.2014.01.002 
Uchida, K., Sumalee, A., and Ho, H. W. 2015. "A stochastic multimodal reliable network design problem under adverse weather conditions." Journal of Advanced Transportation 49: 73-95. doi: https://doi.org/10.1002/atr.1266

Wang, J. Y. T., Ehrgott, M., and Chen, A. 2014. “A bi-objective user equilibrium model of travel time reliability in a road network." Transportation Research Part B: Methodological 66: 4-15. doi: https://doi.org/10.1016/j.trb.2013.10.007

Watling, D. 2006. "User equilibrium traffic network assignment with stochastic travel times and late arrival penalty." European Journal of Operational Research 175: 1539-1556. doi: https://doi.org/10.1016/j.ejor.2005.02.039

Xu, X., Chen, A., Jansuwan, S., Yang, C., and Ryu, S. 2018. "Transportation network redundancy: Complementary measures and computational methods." Transportation Research Part B: Methodological, 114: 68-85. doi: https://doi.org/10.1016/j.trb.2018.05.014

\section{Appendix A}

We summarize the notation that is used in the body part of this paper in Table A1.

\section{Table A1}

$W \quad$ The set of all OD pairs

$w \quad$ OD pair $w, w \in W$

$Q_{w} \quad$ Traffic demand for OD pair $w$

$q_{w} \quad$ Mean of traffic demand for OD pair $w$

$c v_{w} \quad$ Coefficient of variation for OD pair $w$

$K_{w} \quad$ The set of all paths serving OD pair $w$

$k \quad$ Path $k, k \in K_{w}$ 
$F_{k}^{w} \quad$ The flow of path $k$ serving OD pair $w$

$f_{k}^{w} \quad$ Mean of path flow of path $k$ of OD pair $w$

A The set of all links

a Link $a, a \in A$

$V_{a} \quad$ The flow of link $a$

$v_{a} \quad$ Mean flow of link $a$

$t_{a}(\cdot) \quad$ The cost function of link $a$

$t_{a}^{0} \quad$ Free travel time of link $a$

$\alpha_{a}, n_{a} \quad$ Parameters of the cost function of link $a$

$c_{a} \quad$ The capacity of link $a$

$\varepsilon_{a} \quad$ Random term of the flow of link $a$

$\pi_{a} \quad$ Risk premium corresponding to the mean of travel time of link $a$

$v_{a}^{0} \quad$ Certainty equilibrium of flow of link $a$

$\hat{\pi}_{a \mid b} \quad$ Risk premium corresponding to the covariance of travel time between link $a$ and link $b$ with respect to link $a$

$T_{a} \quad$ Travel time of link $a$

$\widehat{\boldsymbol{\pi}}_{a, b} \quad$ Pair of risk premiums corresponding to the covariance of travel time between link $a$ and link $b$ 
$\boldsymbol{\Sigma}_{\mathbf{T}} \quad$ Variance-covariance matrix of link travel time

$\boldsymbol{\Sigma}_{\mathbf{V}} \quad$ Variance-covariance matrix of link flow

$p_{w}^{k} \quad$ Path choice probability of path $k$ serving OD pair $w$

$\eta_{w}^{k} \quad$ Cost of path $k$ serving OD pair $w$

\section{Appendix B}

In this appendix, we show the formulation derived when a method of Duncan (1977) is applied to our network problem. In Duncan (1977), a set of bi-variate risk premiums corresponding to the covariance of link travel time is specified by directly solving (27). By introducing the Moore-Penrose generalized inverse matrix of (23), we could define a specific set of bi-variate risk premiums shown as

$$
\widehat{\boldsymbol{\pi}}_{a, b}=\frac{1}{2}\left(\mathbf{u}_{a, b}^{T}\right)^{-} \operatorname{tr}\left[\mathbf{U}_{a, b} \boldsymbol{\Sigma}_{a, b}\right] \quad \forall a \in A, b \in A
$$

$\left(\mathbf{u}_{a, b}^{T}\right)^{-}$is the generalized inverse matrix of $\mathbf{u}_{a, b}^{T}$ given by

$$
\left(\mathbf{u}_{a, b}^{T}\right)^{-}=\frac{\mathbf{u}_{a, b}}{\left\|\mathbf{u}_{a, b}\right\|^{2}} \quad \forall a \in A, b \in A
$$

Note that "tr" in (B1) implies trace operation and that $\mathbf{u}_{a, b}$ is not a zero vector. The bivariate risk premiums shown by (B1) are those whose secondary norm is minimized. For each pair of two links ( $a, b \in A)$, bi-variate risk premiums (B1) are defined. By collecting the first element of each bi-variate risk premiums vector shown by (B1), a $|A| \times|A|$ matrix 
$\boldsymbol{\Pi}_{1}$ can be defined. Similarly, by collecting the second element of each bi-variate risk premiums vector shown by (B1), a $|A| \times|A|$ matrix, $\boldsymbol{\Pi}_{2}$ can be also defined. Two matrices, $\boldsymbol{\Pi}_{1}$ and $\boldsymbol{\Pi}_{2}$ are respectively shown by

$$
\Pi_{1}=\left(\begin{array}{ccc}
\hat{\pi}_{1 \mid 1} & \cdots & \hat{\pi}_{1|| A \mid} \\
\vdots & \ddots & \vdots \\
\hat{\pi}_{|A| \mid 1} & \cdots & \hat{\pi}_{|A||| A \mid}
\end{array}\right), \Pi_{2}=\left(\begin{array}{ccc}
\hat{\pi}_{1 \mid 1} & \cdots & \hat{\pi}_{|A| \mid 1} \\
\vdots & \ddots & \vdots \\
\hat{\pi}_{1|| A \mid} & \cdots & \hat{\pi}_{|A||| A \mid}
\end{array}\right)\left(=\Pi_{1}^{T}\right)
$$

\section{Appendix C}

Mean of total travel time in the whole road network is given by

$$
E[T T]=\sum_{a \in A} E\left[T_{a}\right]=\sum_{a \in A} t_{a}\left(v_{a}+\pi_{a}\right)
$$

Note that, in this case, the total travel time implies the summation of travel times of all links in the whole road network. The slight change of $\mathrm{E}[\mathrm{TT}]$, when $v_{a}$ changes by $\Delta v_{a}(\forall a \in A)$ is given by

$$
\Delta E[T T]=\sum_{a \in A} e_{a} \cdot \Delta v_{a}
$$

where $e_{a}$ is the sensitivity of $\mathrm{E}[T T]$ with respect to $v_{a}$ given by

$$
\begin{aligned}
e_{a} & =\frac{\partial t_{a}\left(v_{a}+\pi_{a}\right)}{\partial v_{a}}=\frac{\partial t_{a}\left(v_{a}+\pi_{a}\right)}{\partial\left(v_{a}+\pi_{a}\right)} \cdot \frac{\partial\left(v_{a}+\pi_{a}\right)}{\partial v_{a}} \\
& =t_{a}^{(1)}\left(v_{a}+\pi_{a}\right) \cdot\left(1+\frac{d \pi_{a}}{d v_{a}}\right) \forall a \in A
\end{aligned}
$$

Note that, from (13), $\frac{d \pi_{a}}{d v_{a}}$ is analytically derived as 


$$
\frac{d \pi_{a}}{d v_{a}}=\frac{d}{d v_{a}}\left(\frac{1}{2} \frac{t_{a}^{(2)}\left(v_{a}\right)}{t_{a}^{(1)}\left(v_{a}\right)} \operatorname{var}\left[V_{a}\right]\right)
$$

Especially when the variance of link travel time is represented as a product of the coefficient of variance of total traffic demand and mean of its link flow (See Tani and Uchida, 2018), $\operatorname{var}\left[V_{a}\right]=\left(c v \cdot v_{a}\right)^{2}, \frac{d \pi_{a}}{d v_{a}}$ is simply represented as

$$
\frac{d \pi_{a}}{d v_{a}}=\frac{1}{2}(n-1) \cdot c v^{2}
$$

A variance of total travel time can be then expressed as

$$
\operatorname{var}[T T]=\mathbf{e} \boldsymbol{\Sigma}_{\mathbf{v}} \mathbf{e}^{T}
$$

where $\boldsymbol{\Sigma}_{v}$ is the variance-covariance matrix of link flows and $\boldsymbol{e}$ is sensitivity vector shown by

$$
\mathbf{e}=\left(e_{1}, \cdots, e_{|A|}\right)
$$

If travel time variability measurement is the standard deviation (or risk) of total travel time, because the risk is a linear homogenous function with respect to the sensitivities, by applying the Euler's homogeneous function theorem, the risk contribution of link flow $v_{a}$ is calculate as

$$
\begin{gathered}
r_{a}=\frac{\partial \sqrt{\operatorname{var}[T T]}}{\partial e_{a}} \cdot e_{a}=\frac{e_{a}}{\sqrt{\operatorname{var}[T T]}} \cdot \sum_{b \in A} e_{a} \cdot \sigma_{a b} \quad \forall a \in A \\
\sum_{a \in A} r_{a}=\sqrt{\operatorname{var}[T T]}
\end{gathered}
$$


where $\sigma_{a b}$ is the covariance of flows on links $a$ and $b$. Similarly, if a travel time variability measurement is a variance of total travel time because the variance of total travel time is a second-order homogenous function with respect to the sensitivities, the variance contribution of link flow $v_{a}$ is given by

$$
\begin{gathered}
s_{a}=\frac{1}{2} \frac{\partial \operatorname{var}[T T]}{\partial e_{a}} \cdot e_{a}=\sum_{b \in A} e_{a} \cdot \sigma_{a b} \quad \forall a \in A \\
\sum_{a \in A} s_{a}=\operatorname{var}[T T]
\end{gathered}
$$

On the other hand, Bayesian estimation by using observed link flows as likelihood can be applied to the network where link flows follow a multivariate normal distribution. In this case, the posterior distribution of mean link flows can follow a multivariate normal distribution if the corresponding precision matrix is known (e.g., Tani et al., 2018). In the following, two methods for estimating the contribution of link flow on total travel time variability are shown.

It is assumed that the posterior distribution of mean link flows vector $\mathbf{v}=$ $\left(v_{1}, \ldots, v_{|A|}\right)$ follows a multivariate normal distribution with the mean vector of $\hat{\mathbf{v}}$ and the variance-covariance matrix of $\widehat{\boldsymbol{\Sigma}}_{v}$, i.e., $\mathbf{v} \sim \operatorname{MVN}\left(\widehat{\mathbf{v}}, \widehat{\boldsymbol{\Sigma}}_{v}\right)$. Consider first the total travel time variance $V T$ in the network which is now a random variable because mean link flows follow a multivariate normal distribution. The sensitivity of mean of the total travel time variance in the network with respect to $\hat{v}_{a}$ is given by

$$
\begin{aligned}
\hat{e}_{a} & =\frac{\partial E[V T]}{\partial v_{a}}=\frac{\partial}{\partial \hat{v}_{a}}\left(\sum_{b \in A} \hat{\sigma}_{a b}\right) \\
& =\frac{\partial}{\partial \hat{v}_{a}}\left(\sum_{b \in A}\left(t_{a}\left(v_{a}+\hat{\pi}_{a \mid b}\right)+t_{a}\left(v_{a}+\pi_{a}\right)\right) \cdot\left(t_{b}\left(v_{b}+\hat{\pi}_{b \mid a}\right)+t_{b}\left(v_{b}+\pi_{b}\right)\right)\right) \forall a \in A
\end{aligned}
$$


where $\hat{v}_{a}=E\left[v_{a}\right]$ and $\hat{\sigma}_{a b}=\operatorname{cov}\left[v_{a}, v_{b}\right]$. For deducting $(\mathrm{C} 10), \frac{\partial \widehat{\pi}_{a \mid b}}{\partial v_{a}}$ and $\frac{\partial \widehat{\pi}_{b \mid a}}{\partial v_{a}}$ need to be defined. The detailed definitions of $\frac{\partial \widehat{\pi}_{a \mid b}}{\partial v_{a}}$ and $\frac{\partial \widehat{\pi}_{b \mid a}}{\partial v_{a}}$ and the way to calculate both values are described in Appendix D. The change in the mean of the total travel time variance $\mathrm{E}[V T]$ when $\hat{v}_{a}$ changes by $\Delta \hat{v}_{a}(\forall a \in A)$ is

$$
\Delta E[V T]=\sum_{a \in A} \hat{e}_{a} \cdot \Delta \hat{v}_{a}
$$

Therefore, the variance of $V T$ can be given by

$$
\operatorname{var}[V T]=\hat{\mathbf{e}} \boldsymbol{\Sigma}_{\mathbf{V}} \hat{\mathbf{e}}^{T}
$$

where $\hat{\boldsymbol{e}}$ is a sensitivity vector shown as

$$
\widehat{\mathbf{e}}=\left(\hat{e}_{1}, \cdots, \hat{e}_{|A|}\right)
$$

If travel time variability measurement is represented as the standard deviation (or risk) of total travel time, because the risk is a linear homogenous function with respect to the sensitivities, by applying the Euler's homogeneous function theorem, the risk contribution of link flow $v_{a}$ is calculate as

$$
\begin{gathered}
\hat{r}_{a}=\frac{\partial \sqrt{\operatorname{var}[V T]}}{\partial \hat{e}_{a}} \cdot \hat{e}_{a}=\frac{\hat{e}_{a}}{\sqrt{\operatorname{var}[V T]}} \cdot \sum_{b \in A} \hat{e}_{a} \cdot \hat{\sigma}_{a b} \quad \forall a \in A \\
\sum_{a \in A} \hat{r}_{a}=\sqrt{\operatorname{var}[V T]}
\end{gathered}
$$

where $\hat{\sigma}_{a b}$ is the covariance of flows on links $a$ and $b$. Similarly, if travel time variability measurement is the variance of total travel time because the variance of total travel time is a second-order homogenous function with respect to the sensitivities, variance contribution of link flow $v_{a}$ is given by 


$$
\begin{gathered}
\hat{s}_{a}=\frac{1}{2} \frac{\partial \operatorname{var}[V T]}{\partial \hat{e}_{a}} \cdot \hat{e}_{a}=\sum_{b \in A} \hat{e}_{a} \cdot \hat{\sigma}_{a b} \quad \forall a \in A \\
\sum_{a \in A} \hat{s}_{a}=\operatorname{var}[V T]
\end{gathered}
$$

\section{Appendix D}

The first partial derivative of the identity of (19) with respect to the mean of link flow $v_{i}(\forall i \in\{a, b\}, \forall a, \mathrm{~b} \in \mathrm{A})$ is shown as

$$
\begin{aligned}
\frac{\partial t_{i}\left(v_{i}+\pi_{i}\right)}{\partial v_{i}} \cdot t_{j}\left(w_{j}\right)+t_{i}\left(v_{i}+\pi_{i}\right) \cdot \frac{\partial t_{j}\left(w_{j}\right)}{\partial w_{j}} \frac{\partial w_{j}}{\partial v_{i}} & \\
& -\frac{\partial t_{i}\left(v_{i}+\hat{\pi}_{i \mid j}\right)}{\partial w_{i}} \frac{\partial w_{i}}{\partial v_{i}} \cdot t_{j}\left(v_{j}+\pi_{j}\right)=0
\end{aligned}
$$

where

$$
\begin{gathered}
w_{i}=v_{i}+\hat{\pi}_{i \mid j}, w_{j}=v_{j}+\hat{\pi}_{j \mid i} \\
\frac{\partial w_{i}}{\partial v_{i}}=1+\frac{\partial \hat{\pi}_{i \mid j}}{\partial v_{i}} \frac{\partial w_{j}}{\partial v_{i}}=\frac{\partial \hat{\pi}_{j \mid i}}{\partial v_{i}} \\
\frac{\partial t_{i}\left(v_{i}+\pi_{i}\right)}{\partial v_{i}}=t_{i}^{(1)}\left(v_{i}+\pi_{i}\right) \cdot\left(1+\frac{\partial \pi_{i}}{\partial v_{i}}\right)
\end{gathered}
$$

The inverse of a link cost function with respect to link flows can be deductive. Just then, $\hat{\pi}_{j \mid i}$ in the above identity can be explicitly deducted as 


$$
\begin{aligned}
\hat{\pi}_{j \mid i} & =t_{j}^{-1}\left(t_{i}\left(v_{i}+\hat{\pi}_{i \mid j}\right) \cdot \frac{t_{j}\left(v_{j}+\pi_{j}\right)}{t_{i}\left(v_{i}+\pi_{i}\right)}\right)-v_{j} \\
& =\left(\frac{c_{j}^{n}}{\alpha}\left(\frac{t_{i}\left(v_{i}+\hat{\pi}_{i \mid j}\right) \cdot \frac{t_{j}\left(v_{j}+\pi_{j}\right)}{t_{i}\left(v_{i}+\pi_{i}\right)}}{t_{0 j}}-1\right)\right)^{1 / n}-v_{j}
\end{aligned}
$$

By a chain rule, the partial derivative of $\widehat{\pi}_{j \mid i}$ with respect to mean link flow is represented as

$$
\frac{\partial \hat{\pi}_{j \mid i}}{\partial v_{i}}=\frac{\partial t_{j}^{-1}(u)}{\partial u} \frac{\partial u}{\partial v_{i}}
$$

where

$$
\begin{gathered}
u=t_{i}\left(v_{i}+\hat{\pi}_{i \mid j}\right) \cdot \frac{t_{j}\left(v_{j}+\pi_{j}\right)}{t_{i}\left(v_{i}+\pi_{i}\right)} \\
\frac{\partial t_{j}^{-1}(u)}{\partial u}=\frac{c_{j}^{n} \cdot t_{0 i}}{t_{0 j}} \cdot\left(\frac{u}{c}\right)^{n}+\left(\frac{c_{j}^{n} \cdot t_{0 i}}{\alpha} \cdot\left(\alpha\left(\frac{u}{c_{j}}\right)^{n}+1\right)-1\right)^{\frac{1}{n-1}} \\
\frac{\partial u}{\partial v_{i}}=t_{i}^{(1)}\left(v_{i}+\hat{\pi}_{i \mid j}\right) \cdot\left(1+\frac{\partial \hat{\pi}_{i \mid j}}{\partial v_{i}}\right) \cdot \frac{t_{j}\left(v_{j}+\pi_{j}\right)}{t_{i}\left(v_{i}+\pi_{i}\right)}+t_{i}\left(v_{i}+\hat{\pi}_{i \mid j}\right) \cdot\left(\frac{t_{j}\left(v_{j}+\pi_{j}\right)}{t_{i}\left(v_{i}+\pi_{i}\right)}\right)^{(1)} \\
t_{i}^{(1)}\left(v_{i}+\hat{\pi}_{i \mid j}\right)=t_{0 i} \cdot \alpha \cdot n \cdot c_{i}^{-n} \cdot\left(v_{i}+\hat{\pi}_{i \mid j}\right)^{n-1}
\end{gathered}
$$




$$
\begin{aligned}
\left(\frac{t_{j}\left(v_{j}+\pi_{j}\right)}{t_{i}\left(v_{i}+\pi_{i}\right)}\right)^{(1)}= & \frac{\partial}{\partial v_{i}}\left(\frac{t_{j}\left(v_{j}+\pi_{j}\right)}{t_{i}\left(v_{i}+\pi_{i}\right)}\right) \\
= & -\frac{t_{j}\left(v_{j}+\pi_{j}\right) \cdot \alpha \cdot n \cdot\left(\frac{v_{i}+\pi_{i}}{c_{i}}\right)^{n}}{t_{0 i} \cdot\left(v_{i}+\pi_{i}\right) \cdot\left(\alpha \cdot\left(\frac{v_{i}+\pi_{i}}{c_{i}}\right)^{n}+1\right)^{2}} \cdot\left(1+\frac{\partial \pi_{i}}{\partial v_{i}}\right)
\end{aligned}
$$

By solving linear simultaneous equation of (D1)-(D5) and (D6)-(D11), partial derivatives of bi-variate risk premiums, $\frac{\partial \widehat{\pi}_{i \mid j}}{\partial v_{i}}$ and $\frac{\partial \widehat{\pi}_{j \mid i}}{\partial v_{i}}$ are calculated. In the same manner of the process shown above, $\frac{\partial \widehat{\pi}_{i \mid j}}{\partial v_{j}}$ and $\frac{\partial \widehat{\pi}_{j \mid i}}{\partial v_{j}}$ are also calculated.

Figure 1. Concept of certainty equivalent of link flow corresponding to mean of link travel time

Figure 2. Travel time based geometrical explanation of (17), (19) and (20)

Figure 3. Traffic flow-based geometrical explanation of (17) and (19)

Figure 4. Specified sets of bi-variate risk premiums

Figure 5. Test network (Nguyen and Dupuis, 1984)

Figure 6. Variance-covariance of link flow, $\boldsymbol{\Sigma}_{\mathbf{v}}$

Figure 7. Variance-covariance of link travel time, $\boldsymbol{\Sigma}_{\mathbf{T}}$

Figure 8. Risk component matrix $\mathbf{X}_{\mathbf{T}}^{+}$

Figure 9. Risk component matrix $\mathbf{X}_{\mathbf{T}}^{-}$

Figure 10. The risk measurement 


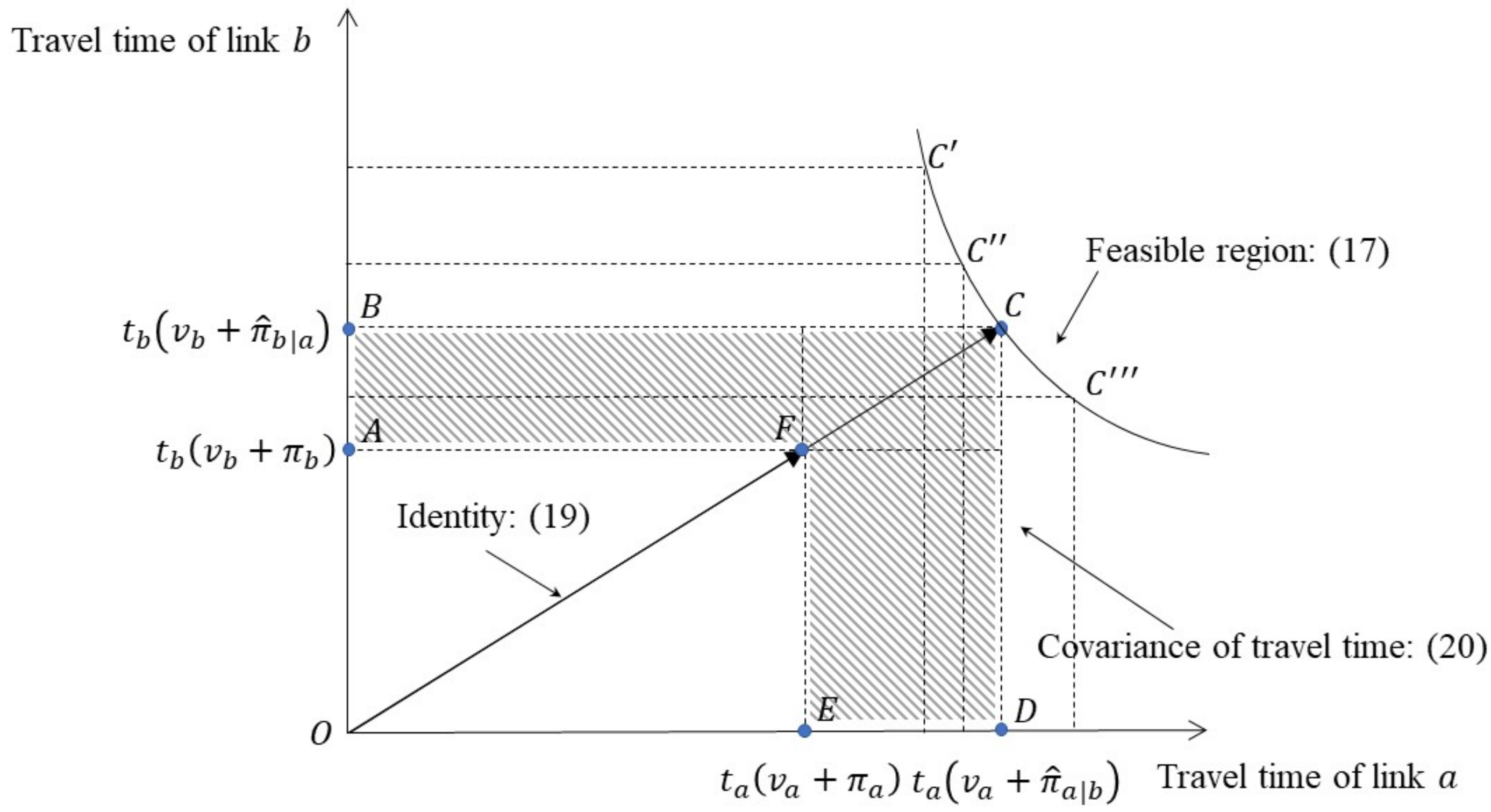




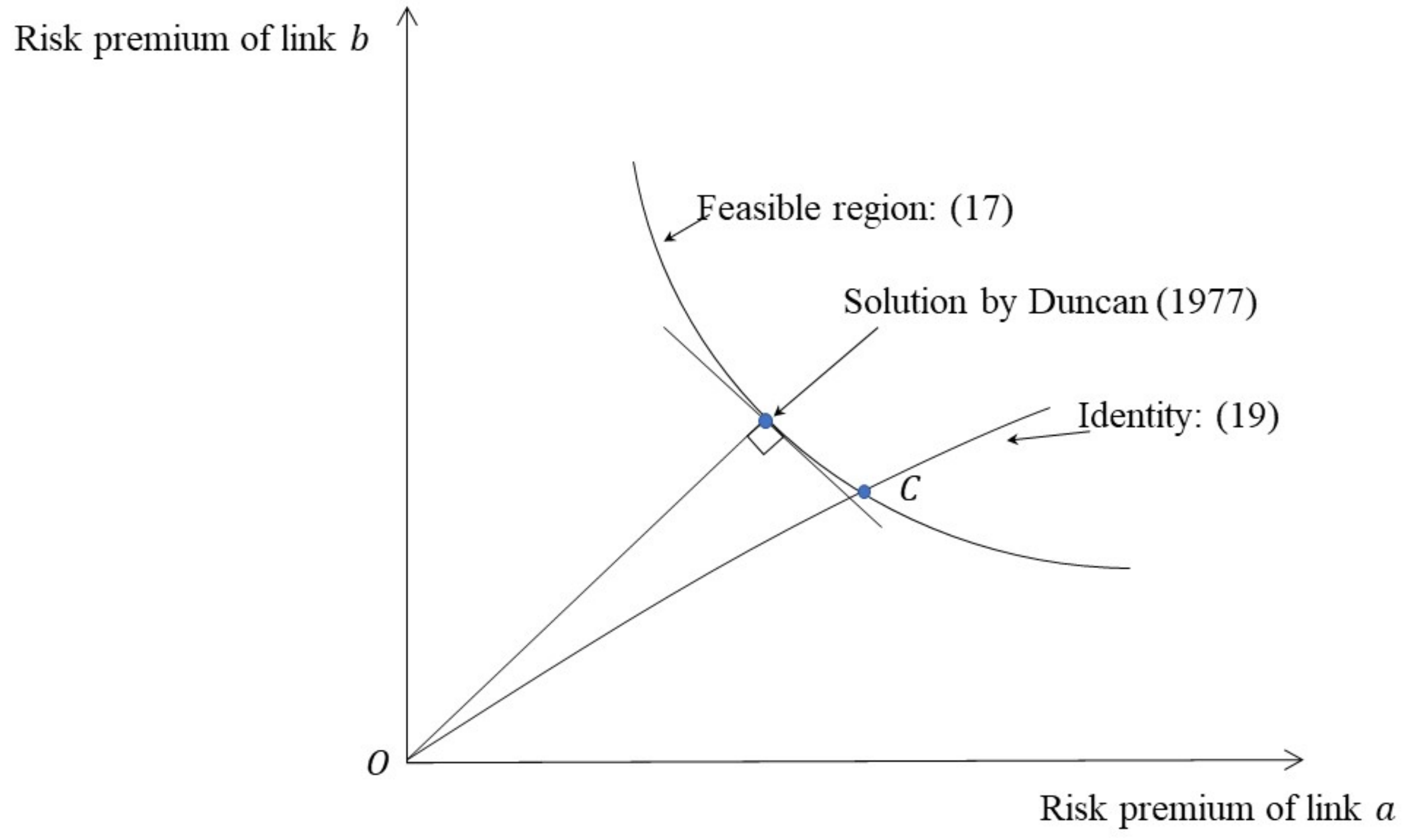




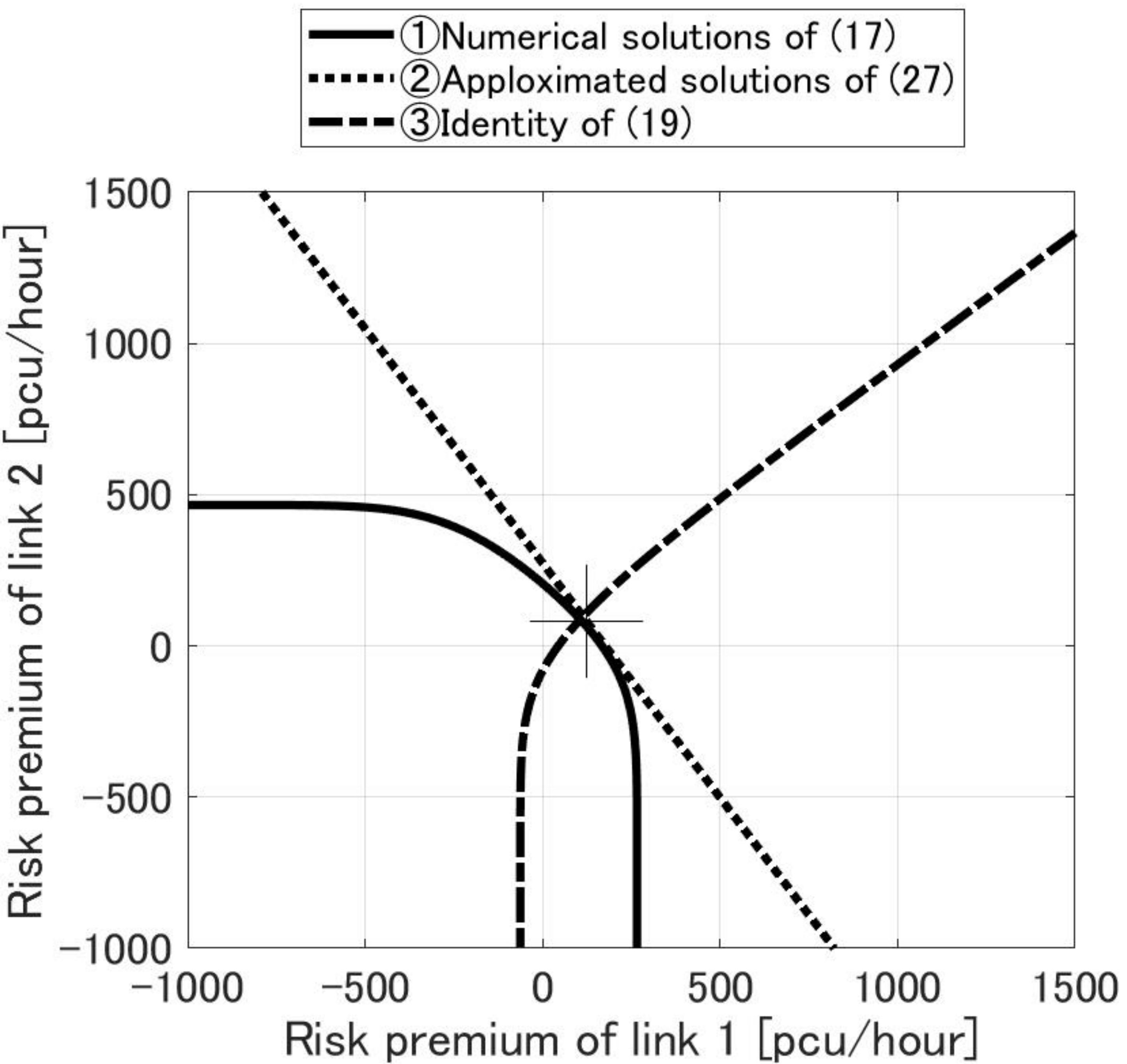




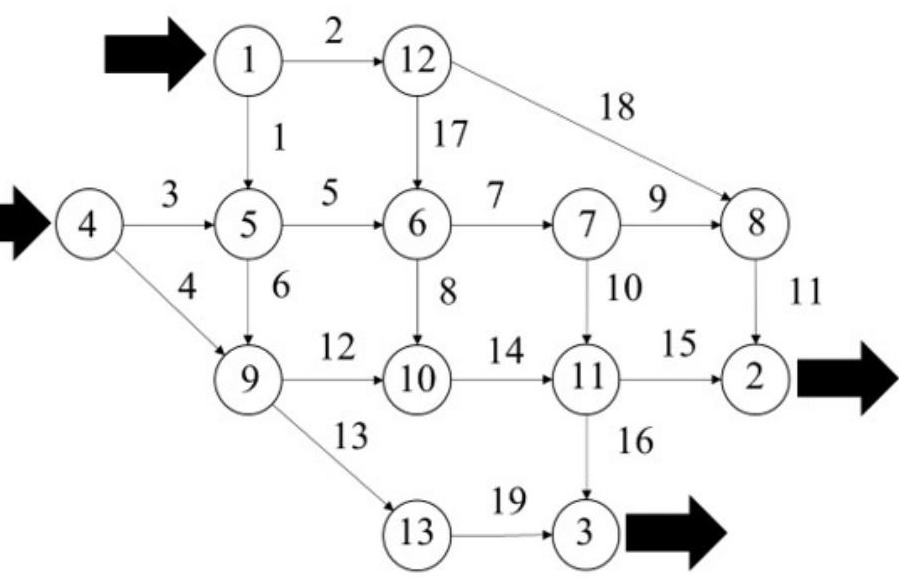





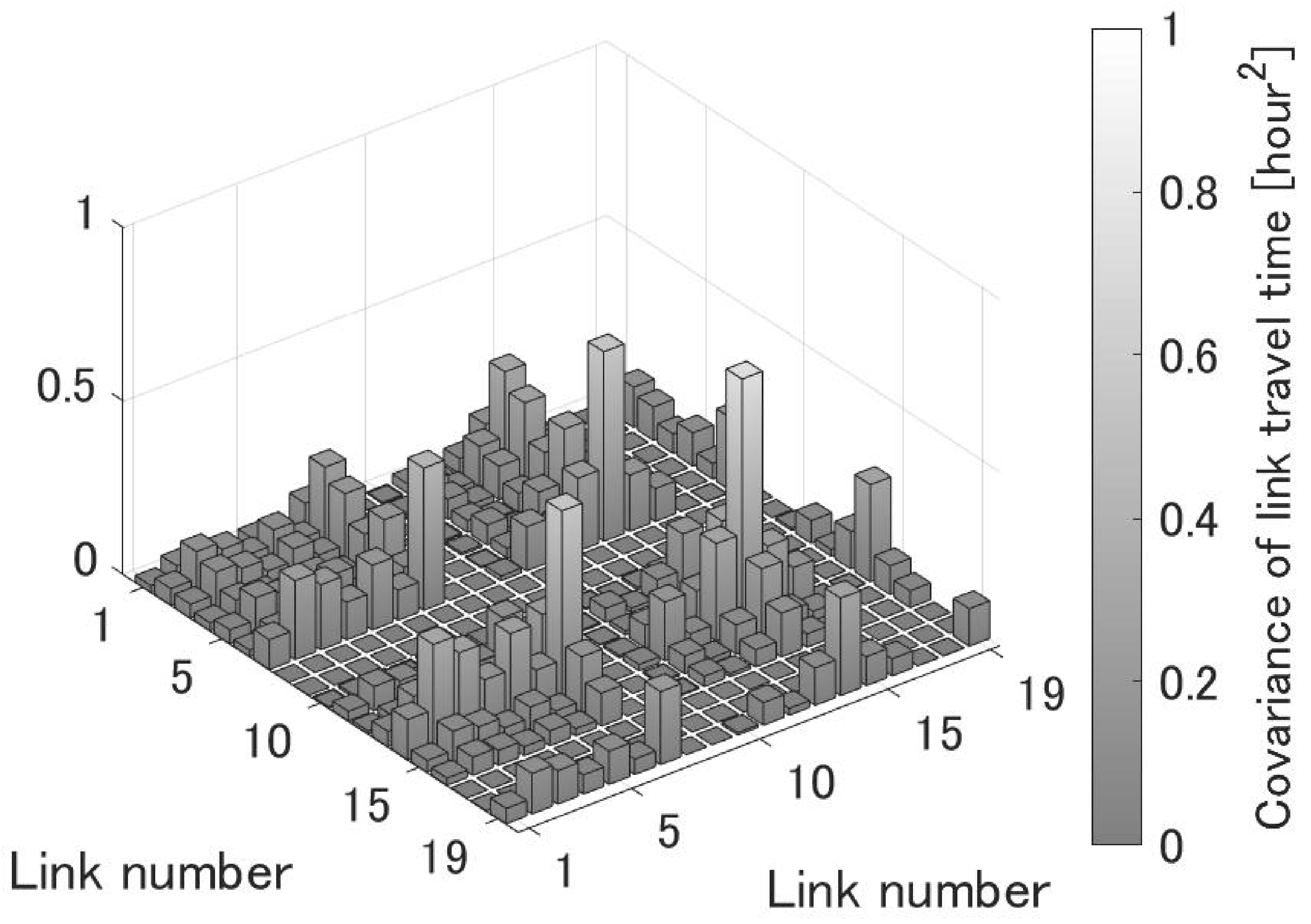




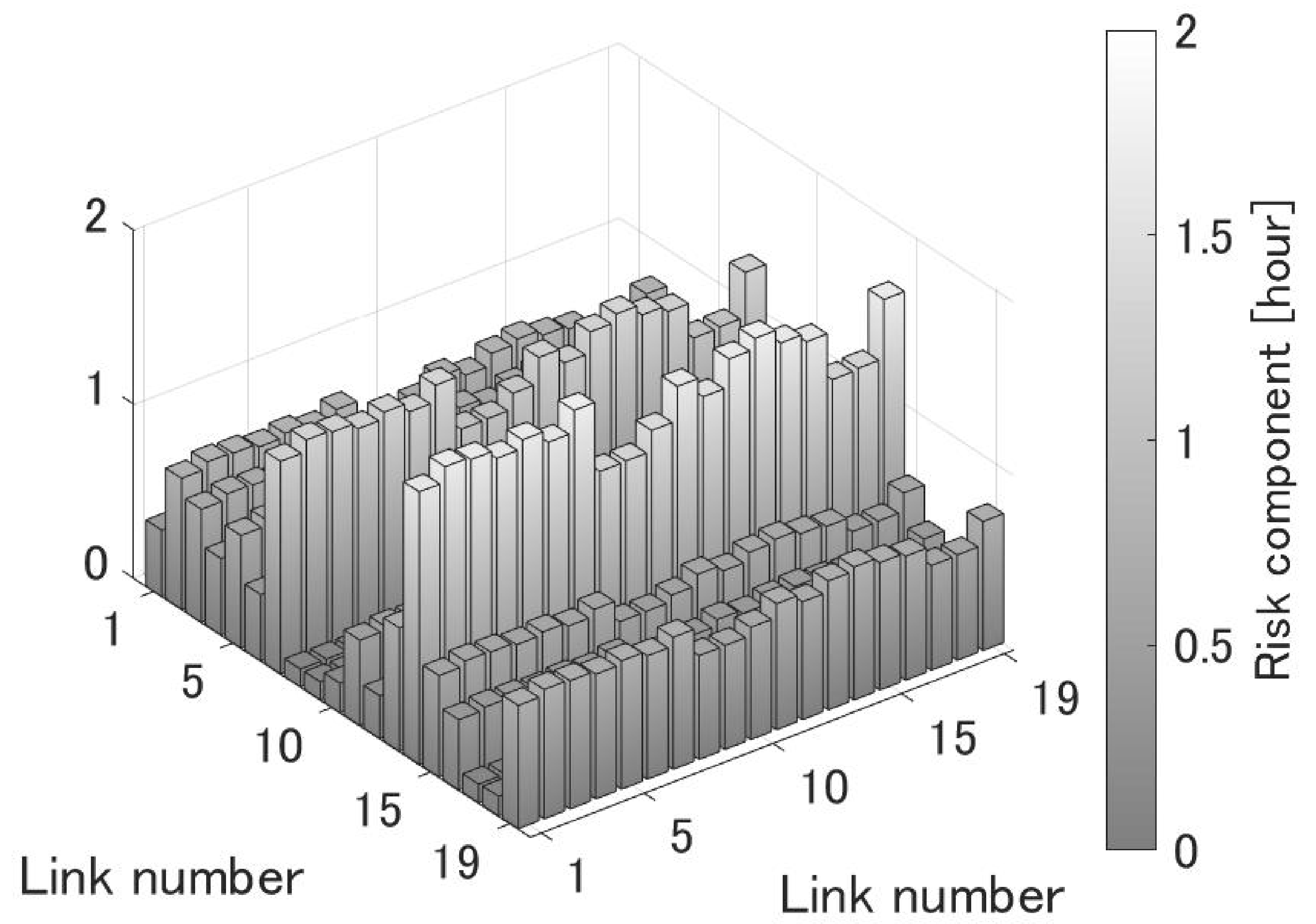




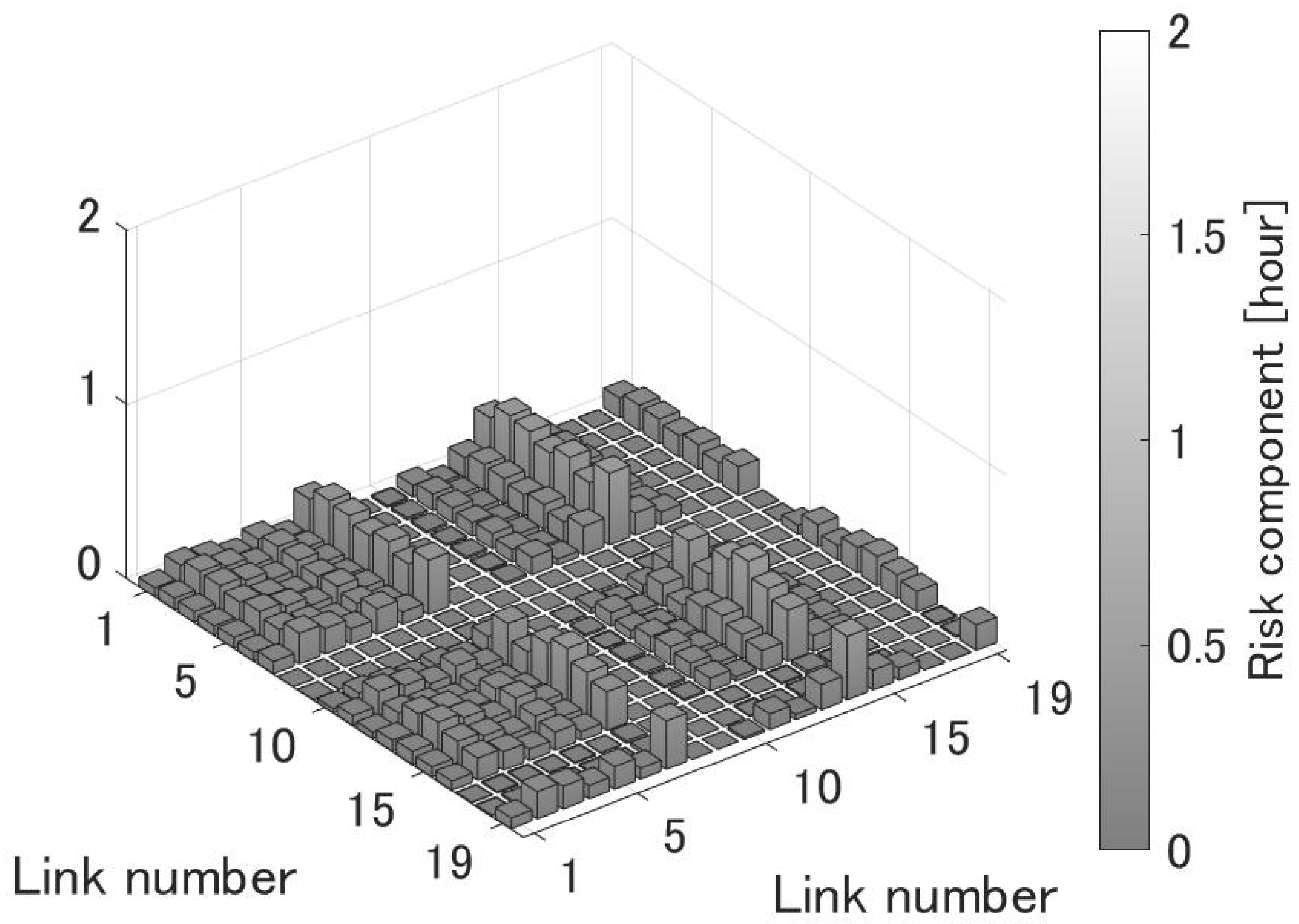




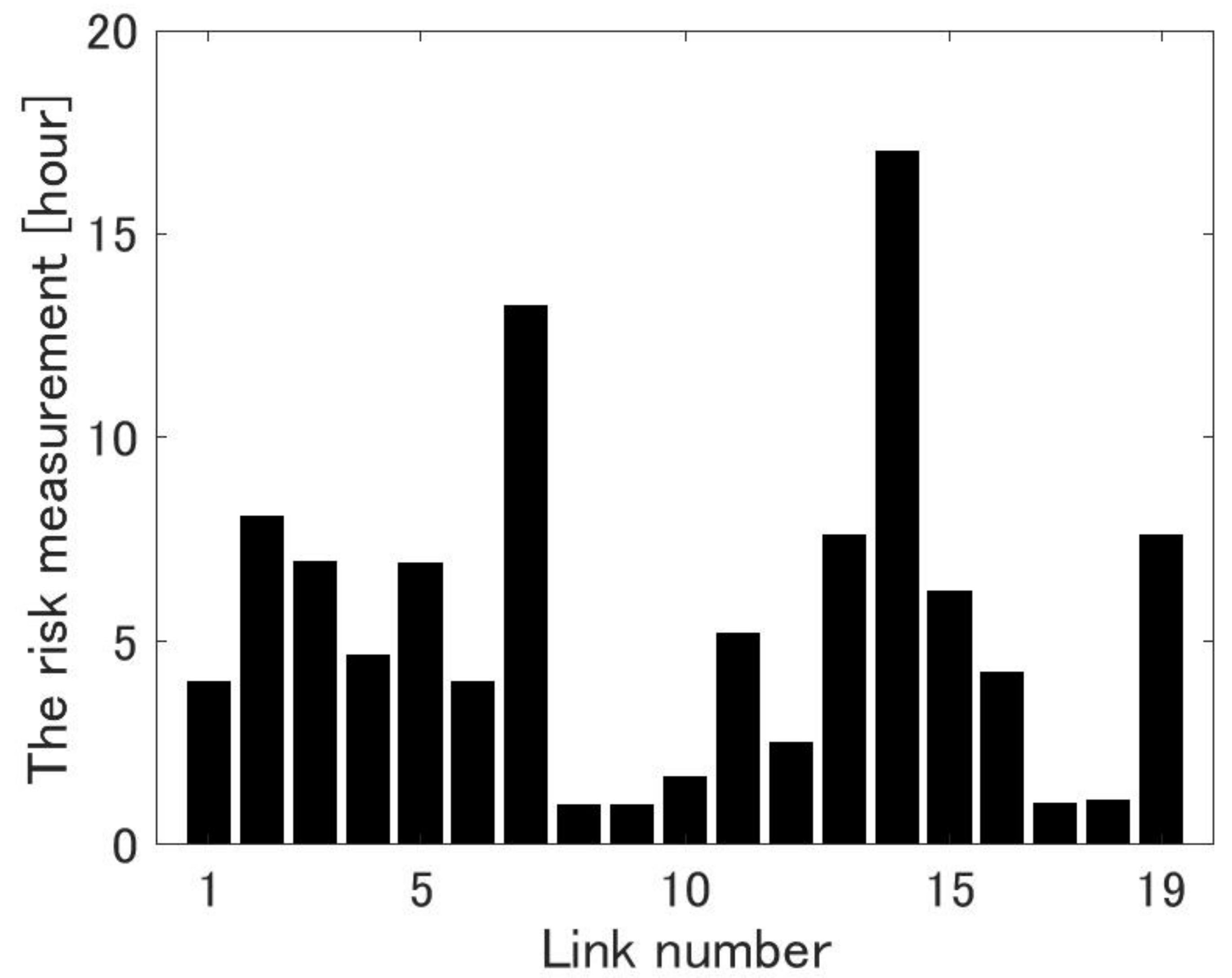

